\title{
Histological and Morphometric Changes in Untraumatised Rabbit Skeletal Muscle TREATED WITH DEEP TRANSVERSE FRICTION.
}

\begin{abstract}
Deep transverse friction (DTF) is used in clinical practice and by its nature it may cause muscle injury. This study investigates the morphologic and morphometric changes in untraumatised rabbit skeletal muscle treated with DTF.

Method: 16 New Zealand white rabbits were studied. The right vastus lateralis muscle was used as a control and the left vastus lateralis was treated with DTF. Muscle biopsies were taken $10 \mathrm{~min}, 24 \mathrm{~h}$ and $48 \mathrm{~h}$ after 1 treatment, $48 \mathrm{~h}$ after 2 treatments and $48 \mathrm{~h}$ and 6 days after 3 treatments. Treatments were $48 \mathrm{~h}$ apart. Biopsies were prepared for light microscopy and tissue morphometry.
\end{abstract}

Results: After 1 DTF treatment, intracellular and extracellular oedema was noted. Contraction bands seen throughout the fibres suggested severe mechanical trauma to the muscle. 48 hours after 1,2, and 3 treatments, the muscle appeared to be recovering with reduced oedema, and the contraction banding was limited to small focal areas throughout each fibre. Six days after the last treatment, the myofibres, although normal in diameter, showed small focal areas of supercontraction and large internalised inclusion bodies composed of a pool of myofilaments or whorls of membranous material. Morphometry showed oedema to be maximal immediately after treatment.

Conclusion: DTF causes a severe but reversible injury to untraumatised myofibres. Its possible mode of action in treatment of injured muscle requires further investigation.

\section{KEY WORDS: DEEP TRANSVERSE FRICTION, SKELETON MUSCLE, LIGHT MICROSCOPY, MORPHOLOGY, MORPHOMETRY}

\section{INTRODUCTION}

Deep transverse frictions (DTF) have been part of the physiotherapists' armamentarium for many years. Despite its acceptance by practitioners there is little scientific evidence to support its use, and to date there has been no systematic review of the efficacy of deep transverse friction treatment. Furthermore, the treatment is painful and has potential to cause further damage.

Menell advocated a specific form of massage, called frictions, in the early

\section{CORRESPONDENCE TO:}

Professor M Mars

Department of Physiology

Nelson R Mandela School of Medicine University of Natal

Private Bag 7, Congella 4013

KwaZulu-Natal

Tel: (031) 260-4364

Fax: (031) 260-4455

Email:mars@nu.ac.za 1900's (Chamberlain 1982). In the mid seventies, Cyriax and Russel popularised the use of a technique called deep transverse friction (DTF) massage, which provides therapeutic movement over a small area (Cyriax and Russel 1977). The potential advantage of DTF is that it allows pressure to be applied at greater depths within muscle and it has been advocated for treatment of muscle strains and tears, tenosynovitis, tendinitis and ligament sprains. A potential disadvantage is that sustained direct pressure on muscle is known to damage skeletal myofibres (Mars and Hadley 1998).

One of the key aims in treatment of soft tissue lesions is to encourage the damaged tissue to regain tensile strength as rapidly as possible (Hunter 1994), with a flexible, functional scar (Dorman 1990). It has been postulated that DTF may achieve these goals through mechanical breakdown of damaged tissue, traumatic hyperaemia and induced analgesia (Winter 1968; Cyriax and
Russel 1977; Cookson and Kent 1979; Chamberlain 1982; Walker 1984; Kushner and Reid 1986). Through the application of force transverse to the plane of the muscle fibres, it was proposed that after injury, deep transverse friction 'spins' or re-aligns the collagen fibres to their correct orientation (Stearns 1940). It has also been suggested that DTF breaks down and reduces transverse and oblique fibrous adhesions that are part of normal scar formation and that DTF can therefore assist in restoring full pain free mobility (Grobbelaar 1991). This concept is not however supported by the histological evidence. In a rabbit model, DTF treated and untreated ligaments were found to have both longitudinal and random arrangement of collagen fibres and it was concluded that DTF's do not have an effect on scar formation (Walker 1984).

According to Norris (1993), the purpose of frictional massage is to promote local hyperaemia, reduce adherent 
scar tissue and induce massage analgesia. It is hypothesised that DTF causes a traumatic reactive hyperaemia on an ischaemic reperfusion basis (Winter 1968; Chamberlain 1982). The increased blood supply to the area may accelerate healing by increasing the supply of nutrients to the injury site and removing metabolites. Again the concept lacks clinical and experimental support, as the reactive hyperaemia will be of relatively short duration. It has been shown that tourniquet induced ischaemia for $90 \mathrm{~min}$ is associated with a reactive hyperaemia that last approximately $15 \mathrm{~min}$ (Mars and Brock-Utne 1991).

It is hypothesised that DTF induced massage analgesia maximally stimulates mechanoreceptors causing the release of neuro-peptides that inhibit and override conduction of pain relayed from nociceptors in slow pain fibres (LeBar et al 1979; de Bruijn 1984).

The histological effects of DTF on skeletal muscle have not been documented. In order to interpret the histological changes that occur following DTF treatment of injured muscle it is necessary to first document the changes produced in untraumatised muscle. The aim of this pilot study was to serially examine the histological and morphometric changes produced by DTF treatment in untraumatised skeletal muscle.

\section{METHODS}

Sixteen New Zealand, white rabbits were studied with the approval of the Ethics and Research Committees of the University of Natal and the University of Durban Westville. The animals were housed in the Biomedical Resource Centre (BRC) of the University of Durban Westville and were maintained under the care of the staff of the BRC. The animals were fed ad libitum and weighed before each treatment session as weight loss may be associated with muscle atrophy.

Before each treatment anaesthesia and analgesia were achieved by an intramuscular injection of a combination of 50\% Ketamine and 50\% Xylazine, $10 \mathrm{mg} / \mathrm{kg}$ of rabbit body weight. The treatment area was the left vastus lateralis at the level of the mid-thigh and the fur was removed with a depilatory to facilitate observation of any inflammatory reaction and to facilitate treatment. To ensure that treatment was applied at the same point, the treatment site was clearly marked with indelible ink.

Treatment consisted of $10 \mathrm{~min}$ of deep transverse friction using the index finger. After treatment the animals were observed during recovery from the anaesthetic and then returned to the holding facility.

Eight animals underwent a single treatment, with muscle biopsies being taken 10 min after treatment $(n=2)$, $24 \mathrm{~h}$ after treatment $(\mathrm{n}=2)$ and $48 \mathrm{~h}$ after treatment $(n=4)$. Four animals underwent 2 treatments, with $48 \mathrm{~h}$ between treatments, and all were biopsied $48 \mathrm{~h}$ after the final treatment. Four animals underwent 3 treatments, with $48 \mathrm{~h}$ between treatments and biopsies were taken $48 \mathrm{~h}$ after the final treatment $(n=2)$ and 6 days after the final treatment $(n=2)$. The timing of the biopsies was based on previous animal studies and was designed to show the spectrum of muscle changes from treatment to repair at 6 days. Control biopsies were taken from the right vastus lateralis muscle in 4 animals.

\section{Muscle Biopsy and Preparation}

Before muscle biopsy, the animals were anaesthetised and then euthanased. The skin was incised longitudinally over treatment site. The fascia was opened and a biopsy of approximately $1 \mathrm{~cm}^{3}$ of vastus lateralis muscle excised from the treatment site. To reduce biopsy induced, "supercontraction" artefact of the muscle, the entire biopsy was immediately immersed in fresh Karnovsky's fixative as per the method of Olmesdahl et al, 1979. After 5 minute immersion, the biopsy was bisected, one segment being immersed in $10 \%$ formal saline, dehydrated through graded ethanols, cleared in xylene and embedded in wax for histology and morphometry. The other segment was minced into approximately $1 \mathrm{~mm}$ cubes and re-immersed in fresh Karnovsky's fixative. Thereafter, the tissue was osmicated in $1 \%$ osmium tetroxide, dehydrated through graded ethanols and embedded in Spurr epoxy resin for high resolution light microscopy.

\section{Light Microscopy \& Morphometry}

Sections of $3 \mu \mathrm{m}$ were cut off the wax embedded tissue with steel blades and thereafter stained for haematoxylin and eosin ( $\mathrm{H}$ and $\mathrm{E}$ ) using a Satura Diversified Stainer. The sections were examined using a light microscope with a 10X objective. The morphometric method used was that described by Mars and Gregory (1991). In brief, suitable areas containing transversely sectioned myofibres were selected for morphometric evaluation. These were then displayed on the monitor of a microcomputer by means of a video camera interfaced with the microscope. The myofibres were considered to be near cylinders and the diameter of transversely sectioned fibres was taken to be the minimum distance across the fibres. This distance was measured for each fibre by positioning two cursors on the muscle fibre image and the computer software calculated and stored the measurement. A minimum of 100 fibre diameters of each specimen was measured in each of the $\mathrm{H}$ and $\mathrm{E}$ stained sections. The mean and standard deviation was determined for the fibres within each animal and for each group of animals. Comparisons of means within and between groups were by analysis of variance with post hoc testing using the Tukey-Kramer test. For intra-group comparisons of groups containing two sets of data, a two tailed unpaired T-test was used. The Chi square test was used to test differences in fibre distribution contingency tables. Significance levels for both tests were set at, $\mathrm{p}<0.05$.

\section{RESULTS}

The average weight of the rabbits prior to the first treatment was $2.74 \pm 0.34 \mathrm{Kg}$ and the average change in weight between treatments or between treatment and biopsy was less than $1 \mathrm{~g}$ with a range of $7 \mathrm{~g}$ gained $(3.7 \%)$ to $9 \mathrm{~g}$ lost $(4.2 \%)$.

There was obvious reddening of the skin of animals immediately after DTF and this was still apparent 24 hours after treatment. The inflammation disappeared within 48 hours of DTF. Following DTF treatment muscle turgor on palpation was increased.

Muscle biopsies taken from control muscles were noted to bleed less than 
Table 1: Mean morphometric data of individual specimens, and the mean and standard deviation of each group of biopsies expressed in $\mathrm{pm}$, from biopsies taken after $1(1 \mathrm{~T}), 2(2 \mathrm{~T})$ and $3(3 \mathrm{~T})$ treatments.

\begin{tabular}{|c|c|c|c|c|c|c|}
\hline Specimen No. & Control & $1 \mathrm{~T}: 10 \mathrm{~min}$ & 1 T: 48 hrs & 2 T: 48 hrs & $3 \mathrm{~T}: 48 \mathrm{hrs}$ & 3 T: 6 days \\
\hline & & Biop $0 \mathrm{hr}$ & Biop $48 \mathrm{hr}$ & Biop $48 \mathrm{hr}$ & Biop $48 \mathrm{hr}$ & Biop 6 day \\
\hline 1 & 56.1 & 64.1 & 58.2 & 51.7 & 58.4 & 56.1 \\
\hline 2 & 55 & 61.8 & 63.2 & 56.4 & 51.7 & 51.4 \\
\hline 3 & 53.6 & & 61 & 48.4 & & \\
\hline 4 & 49.4 & & 58.7 & 59.8 & & \\
\hline Mean & 53.5 & 63.0 & 60.3 & 54.1 & 55.05 & 53.8 \\
\hline SD & 2.9 & 1.6 & 2.3 & 2.3 & 4.7 & 3.3 \\
\hline Max. & 56.1 & 64.1 & 63.2 & 59.8 & 58.4 & 56.1 \\
\hline Min. & 49.4 & 61.8 & 58.2 & 48.4 & 51.7 & 51.4 \\
\hline
\end{tabular}

Table 2: The distribution of skeletal myofibres based on myofibre diameter, after various periods of treatment, expressed as a percentage, and as the mean, standard deviation (SD), and maximum and minimum diameters.

\begin{tabular}{|c|c|c|c|c|c|c|}
\hline Fibre Group & Control & $1 \mathrm{~T}-10 \mathrm{~min}$ & $1 \mathrm{~T}-48 \mathrm{~h}$ & $2 T-48 h$ & $3 \mathrm{~T}-48 \mathrm{~h}$ & $3 T$ - 6days \\
\hline & & Biop $0 \mathrm{hr}$ & Biop $48 \mathrm{hr}$ & Biop $48 \mathrm{hr}$ & Biop $48 \mathrm{hr}$ & Biop 6 day \\
\hline$<20 \mu \mathrm{m}$ & 0.6 & 1 & 0.5 & 0.7 & 0 & 0.5 \\
\hline $21-30 \mu \mathrm{m}$ & 8 & 4 & 4 & 5 & 5 & 8 \\
\hline $31-40 \mu \mathrm{m}$ & 14 & 7 & 11 & 18 & 14 & 15 \\
\hline $41-50 \mu \mathrm{m}$ & 21 & 13 & 18 & 21 & 23 & 24 \\
\hline $51 \cdot 60 \mu \mathrm{m}$ & 22 & 18 & 18 & 26 & 27 & 22 \\
\hline $61-70 \mu \mathrm{m}$ & 20 & 24 & 18 & 15 & 14 & 12 \\
\hline $71-80 \mu \mathrm{m}$ & 9 & 15 & 17 & 12 & 12 & 9 \\
\hline $81-90 \mu \mathrm{m}$ & 4 & 10 & 9 & 3 & 6 & 7 \\
\hline $91-100 \mu \mathrm{m}$ & 1 & 7 & 3 & 0.3 & 0 & 0.5 \\
\hline $101-110 \mu \mathrm{m}$ & 0.5 & 1 & 2 & 0 & 0 & 0.5 \\
\hline$>110 \mu \mathrm{m}$ & 0 & 0.5 & 1 & 0 & 0 & 1 \\
\hline Mean $(\mu \mathrm{m})$ & 53.9 & 62.8 & 60.5 & 53.4 & 54.7 & 53.8 \\
\hline$S D(\mu \mathrm{m})$ & 16.4 & 18.8 & 19.1 & 15.3 & 15.2 & 18.1 \\
\hline High $(\mu \mathrm{m})$ & 108 & 121 & 131 & 100 & 87 & 113 \\
\hline Low $(\mu \mathrm{m})$ & 16 & 18 & 20 & 18 & 23 & 17 \\
\hline No Fibres & 341 & 217 & 343 & 413 & 160 & 200 \\
\hline No of Specs. & 4 & 2 & 4 & 4 & 2 & 2 \\
\hline
\end{tabular}

DTF treated muscle. Muscle biopsied immediately after a single treatment appeared to be darker in colour, consistent with local haemorrhage.

\section{Histology and Morphometry}

Myofibres in control tissue appeared as irregular polygons grouped into bundles (Figure 1). The morphometric data of individual specimens from each DTF therapy group is shown in table 1 . Note that in control specimens, all means were within the range $49.4 \mu \mathrm{m}$ to $56.1 \mu \mathrm{m}$. In both groups receiving 1 DTF treatment
(IT) and biopsied $10 \mathrm{~min}$ and $48 \mathrm{~h}$ after treatment, the means of all specimens were greater than control values. In the case of 2T: 48 hours and 3T: 48 hours, while group mean values were within the normal range $(54.1 \mu \mathrm{m}$ and $55.05 \mu \mathrm{m}$ respectively), $50 \%$ of the specimens in each group had means above normal values. Six days after 3 DTF treatments, the diameters of myofibres in both specimens were within normal values. The mean morphometric data of the individual samples are shown in Table 2.

Fibre diameters in controls and all treatment groups ranged from $16 \mu \mathrm{m}$ to $131 \mu \mathrm{m}$. Significant differences in the distribution of fibre diameters, $(\mathrm{p}<0.05)$ were shown between controls and biopsies taken $10 \mathrm{~min}$ and $48 \mathrm{~h}$, after a single treatment (Figure 2). The distribution of fibre diameters was similar to the controls. Comparison of mean diameters by ANOVA shows a significant difference $(p=0.038)$ with the greatest difference between the controls and the biopsies taken immediately after one treatment. However, post hoc testing did not reveal significant differences between groups. 
Figure 1: Light micrograph of $5 \mathrm{\mu m}$ wax embedded, haematoxylin and eosin stained section showing cross-sectioned myofibres (F) in normal rabbit skeletal muscle. The arrows mark the diameters of fibres used for morphometry.

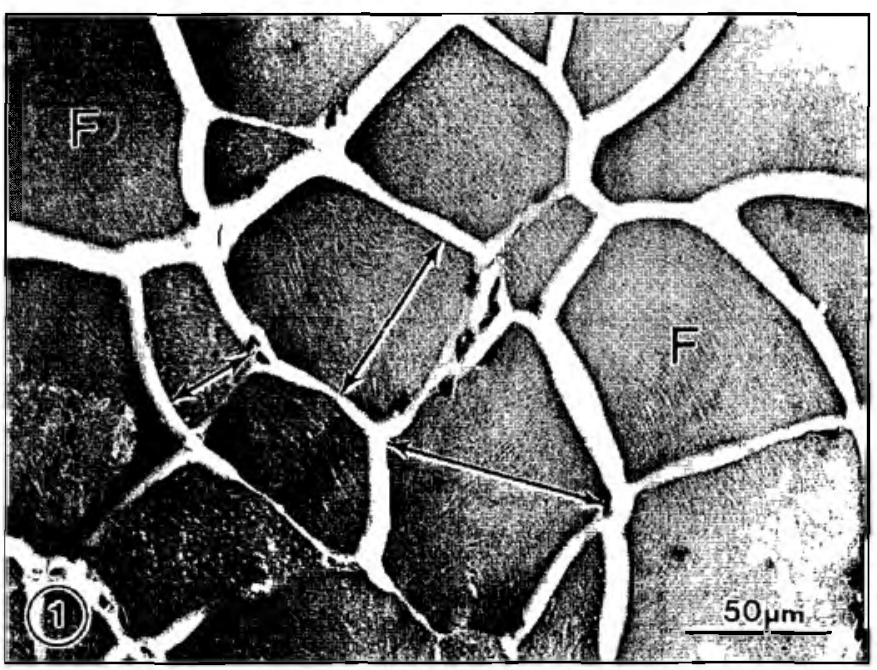

Figure 3: 10 minutes after 1 DTF: LM of 1 (m toluidine blue stained, resin embedded section showing numerous supercontraction bands $(C)$ through the full thickness of the muscle fibre. $M=$ myofibrils.

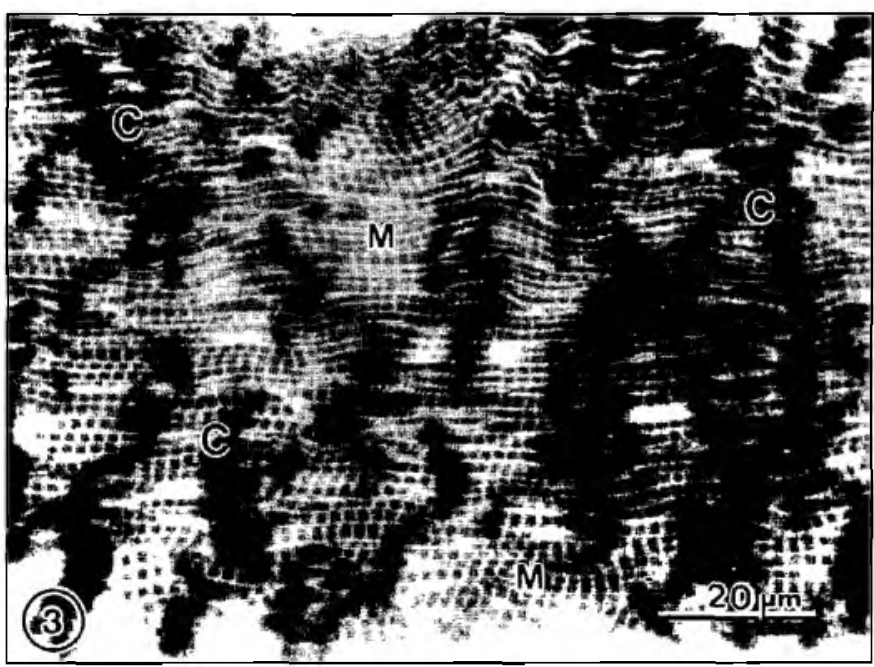

Figure 2: Bar histogram showing the distribution of fibre diameters in control muscle and tissue, 10 minutes and 48 hours after 1 DTF treatment. Note shift of myofibres to larger diameters after 1 DTF.

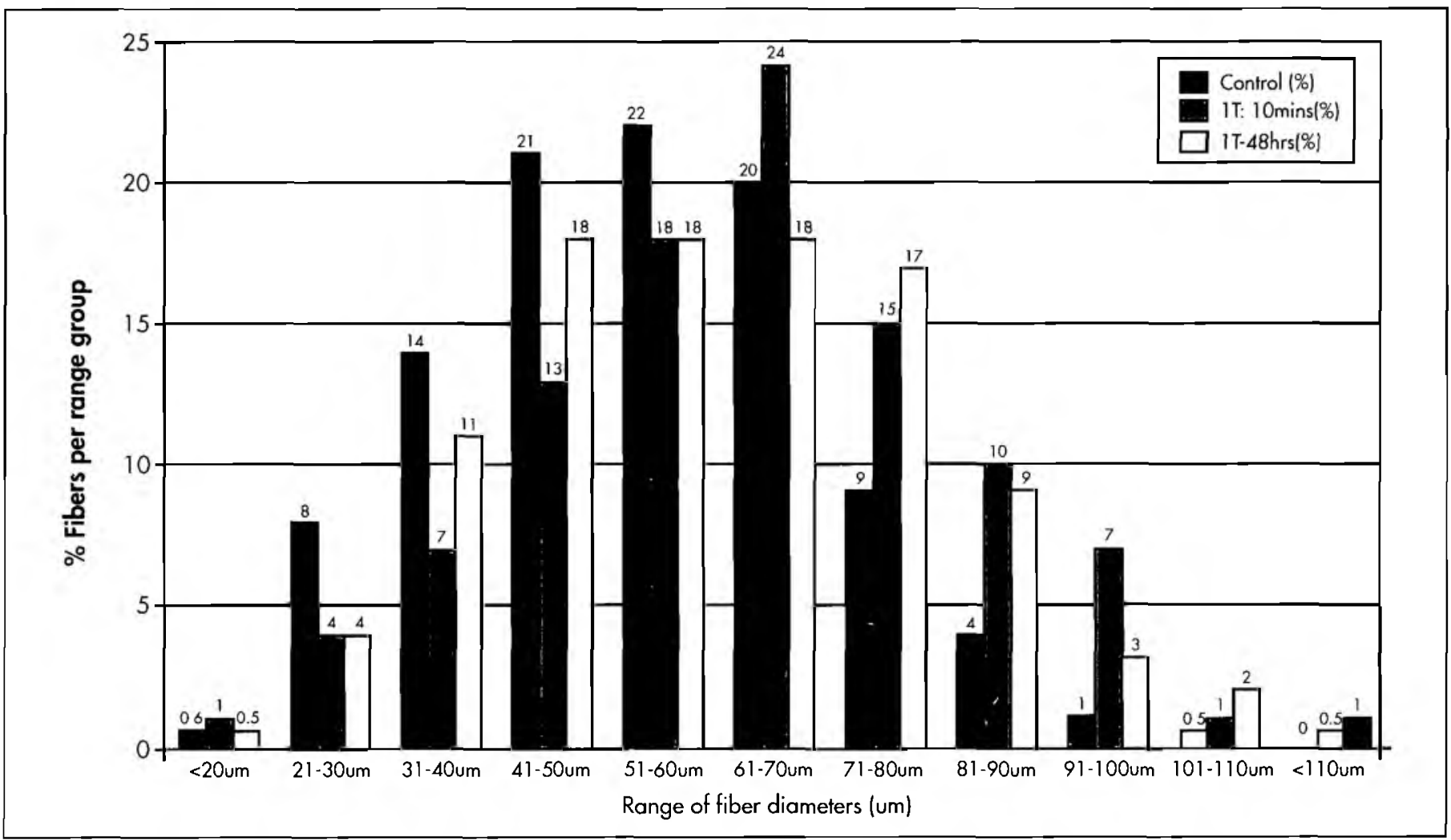

Light Microscopy of 1 pm

Toluidine Blue Sections

The tissue was orientated and sectioned in such a manner as to enable the longitudinal aspects of myofibres to be examined. Control specimens showed myofibres with regularly arranged myofibrils with peripheral, elongated oval nuclei. There was no evidence of intercellular or intracellular oedema and there were no inflammatory cells in the interfibre spaces. Ten minutes after 1 treatment, numerous densely staining, super-contraction bands were observed extending vertically through the diameter of most myofibres (Figure 3). In some instances, myofibrils appeared to have separated from one another and had become disorientated. The fibres were separated by larger spaces suggesting intercellular oedema.

There were no obvious differences in the morphology of myofibres in specimens taken 48 hours after 1, 2 or 3 DTF treatments. In all cases the contractions, while still present, were smaller and restricted to a few myofibrils, especially those beneath the sarcolemma (Figure 4). The myofibres appeared less separated, 48 hours after each treatment. Six days after 3 treatments, there was minimal evidence of contraction banding which, when present, was exclusively 
Figure 4: 48 hours after 1 DTF: LM of 1 ( $\mathrm{m}$, toluidine blue stained, resin embedded section showing occasional peripheral and internal contraction bands involving up to 4 sarcomeres (arrowed). $\mathbf{N}$ = nucleus.

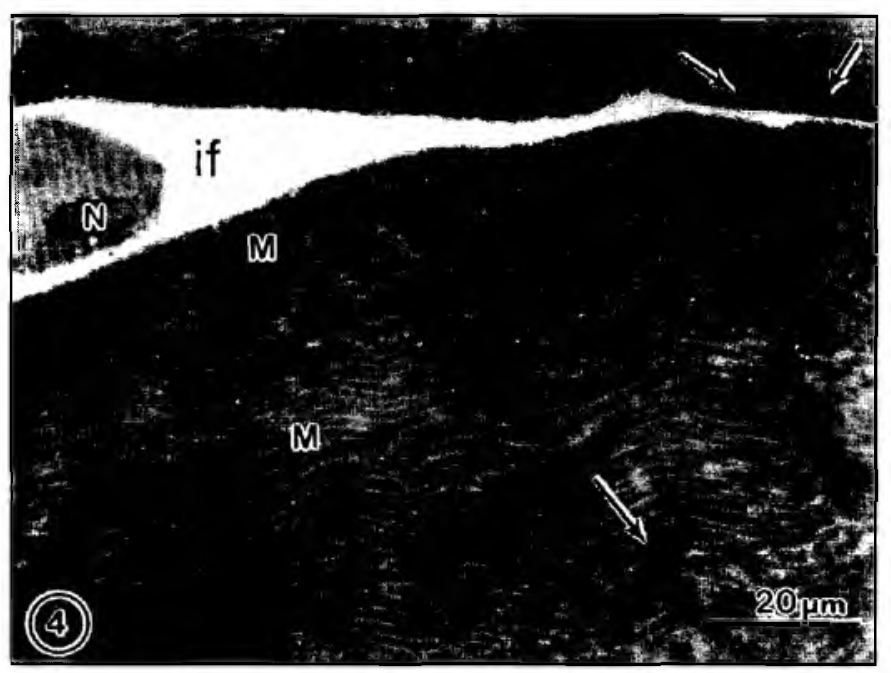

Figure 5: 6 days after 3 DTF: LM of $1 \mathrm{~lm}$, tolvidine blue stained, resin embedded section showing large, amorphous inclusions within the fibre (AIB and arrows). Note absence of contraction bands in these fibres.

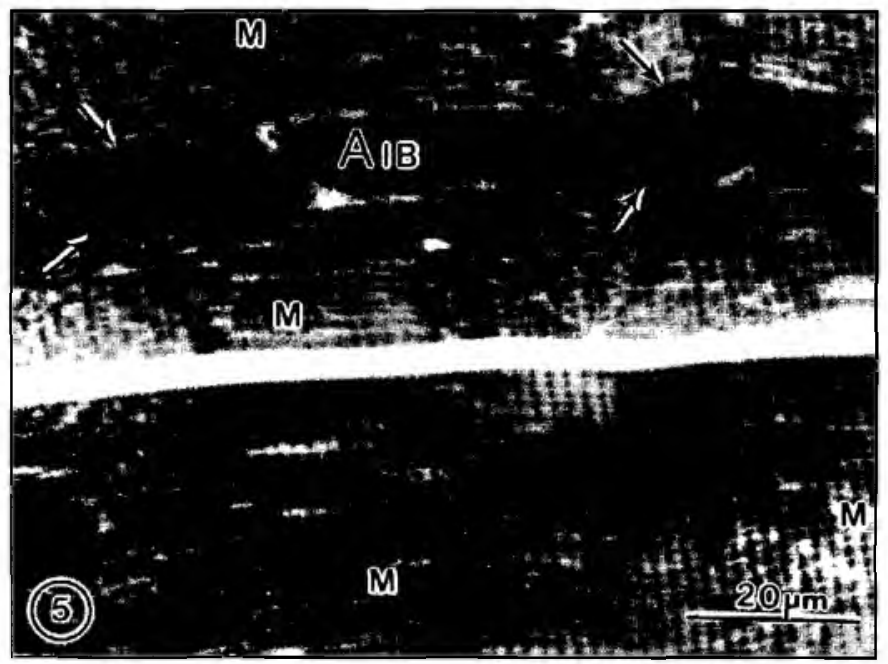

Figure 6: 6 days after 3 DTF: LM of $11 \mathrm{~m}$, toluidine blue stained, resin embedded section showing large inclusion bodies comprised of membranous whorls (MIB and arrows). Note the absence of contraction bands in the fibre.

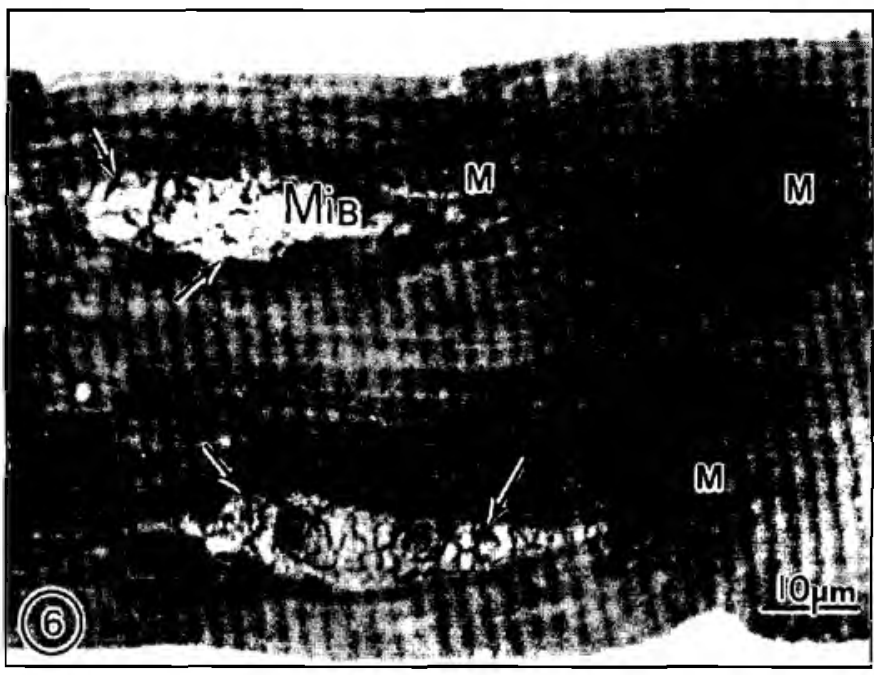

restricted to the periphery. The most striking anomaly was the presence of large inclusion bodies near the centre of fibres. Some were particularly toluidine blue positive while others were pale and appeared to contain filamentous structures (Figures 5 and 6). Intercellular spaces had returned to those seen in control specimens.

\section{DISCUSSION}

The morphometric and histological results show that DTF causes myofibre oedema. Shortly after treatment, myofibres had increased in diameter and although not quantified, there appeared to be an increase in interfibre spaces. There were, however, some anomalies in the results from the groups of specimens taken 48 hours after DTF. While there was a non-significant increase in group mean fibre diameters (GMD) 48 hours after 1 treatment, there were no apparent differences in GMD 48 hours after 2 and 3 DTF treatments. Closer examination of the data from each specimen showed that $50 \%$ of the samples in each group were larger than those in the normal range (Table 1). There is a reduction in myofibre oedema 48 hours after DTF, and 6 days post DTF the myofibres have returned to control dimensions.

Control muscle appeared normal when viewed by light microscopy. No contraction bands were present, indicating that the preparation of tissue after the biopsy procedure had not caused mechanically induced contraction bands (Olmesdahl et al 1979). The morphological results showed that DTF, caused rapid change in myofibre structure. Shortly after DTF, the myofibres exhibited severe supercontractions throughout the full length of each cell. In addition, there was evidence of myofibril disorganisation and displacement. Irrespective of the number of DTF treatments, 48 hours after treatment supercontractions were reduced and limited to focal areas, especially near the periphery of fibres. Six days after DTF, supercontractions were limited to occasional groups of supercontracted sarcomeres on the periphery of fibres. However, of particular interest were the two types of inclusion seen in many fibres.

The first type of inclusion appeared pale in toluidine blue stained sections and contained what appeared to be membranous fragments, while the second type were darkly stained and appeared to contain myofibrillar elements (actin and myosin filaments). These are particularly significant observations indicating that DTF causes a serious, albeit reversible injury to skeletal myofibres. No other abnormalities of the myofibres were noted.

Muscle injury is caused in many ways and expressed by many and varied morphological changes within myofibres and connective tissue. The changes caused by controlled injury have been described in several animal models and are considered to reflect injury and reparative processes in man. Stretch-induced injuries in rabbit skeletal muscle are marked by "disruption" and haemorrhage within the muscle (Nikolaou et al 1987). Friden et al (1983a) showed that there was microscopically identifiable disruption of myofibrils and streaming of $Z$ line in injured human muscle. In another study, electron microscopy showed significant changes in the sarcomeres of overstrained vastus lateralis muscle in cyclists (Friden et al 1983b). 
In a rabbit model, Reddy et al (1991) found that 1 hour after injury "torn" fibres had supercontracted sarcomeres within $100 \mu \mathrm{m}$ of the rupture site, both proximal and distal to the injury. Sarcomere length gradually increased with distance from the rupture site with normalised at a distance of approximately $500 \mu \mathrm{m}$. Supercontraction is a common feature of muscle injury and is thought to be a consequence of massive calcium release from the sarcoplasmic reticulum or influx of calcium through the sarcolemma.

The following is a postulate of how DTF may encourage muscle healing. Mechanical stimuli have been shown to alter cellular functions including ion transport and protein synthesis (Schwartz et al 1991). 1t is suggested that following DTF, there is a significant increase in blood flow to the muscle (Winter 1968; Chamberlain 1982; de Bruijn 1984; Walker 1984; Kushner and Reid 1986; Norris 1993). In addition to hyperaemia, this study shows that DTF causes a reversible muscle injury, which initiates an inflammatory response expressed in the form of muscle and myofibre oedema and myofibre supercontraction. That DTF causes myofibre oedema has been reported by Prentice and Bell (1990) who suggest that "massage appears to facilitate tissue repair by inducing and reducing oedema through the pressure that provides the initial stimulus for the healing cascade". Perhaps this additional injury to already damaged muscle increases the tissue cytokine response and thus the "natural" inflammatory response, thereby attracting the necessary phagocytes, other inflammatory cells to the damaged area. Concentrating these moieties at the site of injury may facilitate rapid healing.

On the other hand DTF may not be enhancing the inflammatory cascade but may be slowing healing by the addition of an iatrogenic injury to already traumatised tissue.

This pilot study shows that DTF alters muscle and myofibre structure at the macro- and microscopic level. There are, however, many unanswered questions. Are the different fibre types affected to the same extent by DTF with regards oedema and pathomorphology? How oedematous does muscle become after DTF and what is the natural time course of the return to normality? What ultrastructural changes occur in myofibres exposed to DTF and what are the inclusion bodies seen 6 days after treatment composed of? Further studies employing histochemistry, morphometry and transmission electron microscopy are required to answer these questions.

\section{CONCLUSION}

The results of this study show that deep transverse friction causes morphological changes in normal, rabbit skeletal muscle fibres. Myofibres become oedematous shortly after DTF and they develop supercontraction bands. These are characteristic of muscle injury. The severity of injury reduces with time showing that injury caused by DTF is reversible. It is postulated that DTF may promote healing by causing an additional inflammatory reaction which triggers a cascade of healing responses thereby shortening healing time. It may also be of no benefit and merely add to the existing injury. Further studies are required to determine the mode of action of deep transverse friction therapy.

\section{ACKNOWLEDGEMENT:}

This study was funded in part by grants from the South African Society of Physiotherapy and the University of Durban Westville.

\section{REFERENCES}

Chamberlain GL 1982 Cyriax's friction massage: a review. Journal of Orthopaedic and Sports Physical Therapy 4:16-22

Cookson J, Kent B 1979 Orthopaedic manual therapy: an overview. Physical Therapy 59: 136-146

Cyriax J, Russel G 1977 Treatment by manipulation massage and injection. In: Textbook of Orthopaedic Medicine, Cyriax J (editor). Williams and Williams, Baltimore: 18-36

De Bruijn R 1984 Deep transverse friction: Its analgesic effect. International Journal of Sports Medicine Suppl 5: 35-36

Dorman P 1990 The significance of scar tissue on rehabilitation of the athlete. Sport and Health 8:17-19

Friden J, Seger J, Sjostrom M, Ekblom B 1983a Adaptive response in human skeletal muscle subjected to prolonged eccentric training. International Journal of Sports Medicine 4:177-183
Friden J, Sjostrom M, Ekblom B 1983b Myofibrillar damage following intense eccentric exercise in man. International Journal of Sports Medicine 4:170-176

Grobbelaar C 1991 The clinical effectiveness of deep transverse friction in the treatment of acute muscle injuries in sports. (Thesis, Cape Town)

Hunter G 1994 Specific soft tissue mobilisations in the treatment of soft tissue lesions, Physiotherapy 80: 15-21

Kushner S, Reid D 1986 Manipulation in the treatment of tennis elbow. Journal of Orthopaedic Sports Physical Therapy 7: 264-272

LeBar D, Rivot JP, Guilband G, Menetrey D, Bessen JM 1979 The depressive effects of morphine on the $\mathrm{C}$ fibre responseof doresal horn neurons pretreated or not by pCPA. Brain Research 176:337-353

Mars M, Brock-Utne JG 1991 The effect of tourniquet release on intra-compartmental pressure in the bandaged and unbandaged limb. Journal of Hand Surgery - British 16: 318-322

Mars M, Gregory MA 1991 A histometric analysis of skeletal myofibres following 90 min of tourniquet ischemia and reperfusion. Journal of Surgical Research 50: 191-195

Mars M, Hadley GP 1998 Raised intracompartmental pressure and compartment syndromes. Injury 29: 403-411

Nikolaou P, MacDonald B, Glisson R, Seaber A, Garrett W 1987 Biochemical and histological evaluation of muscle after controlled strain injury. American Journal of Sports Medicine 15:9-14

Norris C 1993 Sports Injuries. Butterworth Heinemann, New York: 109-111

Olmesdahl PJ, Gregory MA, Cameron EWJ 1979 Ultrastructural artefacts in biopsied normal myocardial biopsy in man. Thorax 34:82-90

Prentice WE, Bell GW 1990 Pathophysiology of musculoskeletal injuries and the healing process. In: Rehabilitation Techniques in Sports Medicine, Times Mirror, St. Louis

Reddy A, Reedy M, Best T 1991 Evaluation of strain injuries in rabbit skeletal muscle using a single fibre model. Surgery Forum 12: 44

Schwartz M, Lechen D, Ingber D 199] Fibronectin activates the NA/H antiport by inducing clustering and immobilisation of its receptors, independent of cell shape. Proceedings of the National Academy of Science 88: 121-122

Stearns M 1940 Studies on the development of connective tissue in transparent chambers in the rabbit's ear. American Journal of Anatomy 67: 55-97

Walker J 1984 Deep transverse frictions in ligament healing. Journal of Orthopaedic Sports Physical Therapy 6:89-94

Winter D 1968 Transverse frictions. Physiotherapy 24:5-7 